\title{
SimBit: A high performance, flexible and easy-to-use population genetic simulator
}

\author{
Remi Matthey-Doret ${ }^{1}$ \\ ${ }^{1}$ University Of British Columbia
}

September 11, 2020

\begin{abstract}
SimBit is a general purpose and high performance forward-in-time population genetics simulator. SimBit has been designed to be able to model a wide diversity of complex scenarios from a simple set of commands that are very flexible. SimBit also comes with a $\mathrm{R}$ wrapper that simplifies the management of an entire research project from the creation of a grid of parameters and corresponding inputs, running simulations and gathering outputs for analysis. Implementing various representations of the individual's genotype allows SimBit to sustain a high performance in a wide diversity of simulation scenarios. SimBit's performance was extensively benchmarked in comparison to SLiM, Nemo and SFS_CODE. No single program systematically outperforms the others but SimBit is most often the highest performing program and maintains high performance in all scenarios considered.
\end{abstract}

\section{Hosted file}

MS.pdf available at https://authorea.com/users/358327/articles/480512-simbit-a-highperformance-flexible-and-easy-to-use-population-genetic-simulator 